\title{
Global mental health intervention research and mass trauma
}

\author{
This article was published in the following Dove Press journal: \\ Open Access Journal of Clinical Trials \\ 13 March 2013 \\ Number of times this article has been viewed
}

\author{
Susan Meffert' \\ Solvig Ekblad ${ }^{2}$ \\ 'Department of Psychiatry, University \\ of California, San Francisco, San \\ Francisco, CA, USA; ${ }^{2}$ Karolinska \\ Institutet, Department of Learning, \\ Informatics, Management and Ethics \\ (LIME), Cultural Medicine Unit, \\ Stockholm, Sweden
}

\begin{abstract}
The impact of mass trauma on mental health and the treatment of resulting disorders has been a major focus of global mental health work since the inauguration of the field. Descriptive studies in the 1990s provided convincing evidence of the importance of addressing global mental health needs in the aftermath of mass trauma. Nonetheless, despite calls to move ahead with interventional research, few studies have tested the effectiveness of the treatments for survivors of mass trauma. In this study, we use a translational science model to review the status of intervention research for adult survivors of mass trauma with the goal of identifying promising treatments, and presenting a logic model for using available data in a manner that is sensitive to community needs, and integrating with existing systems for capacity building.
\end{abstract}

Keywords: global mental health, trauma, disaster, PTSD

\section{Introduction}

\section{Global mental health scale-up and translational research}

Over the past decade, many have articulated the pressing need for global mental health care in low- and middle-income countries (LMICs). ${ }^{1-6}$ In 1996, the World Health Organization's (WHO) Global Burden of Disease (GBD) study provided the first epidemiological data that identified the presence of mental illness in LMICs. The GBD study found that the burden of mental illness was not only higher than many diseases traditionally thought to dominate poor health in LMICs, but that mental illness was the number one cause of disability worldwide. ${ }^{2,3}$

Despite our growing understanding of the descriptive statistics of mental disorders in LMICs, there continues to be such a dearth of mental health care intervention research that the Lancet Mental Health Group issued a "Call to Action" in 2007 in an effort to rally the development of professionalized global mental health care. ${ }^{1}$ In 2009 , the WHO reiterated the urgent need to develop effective mental health care interventions and delivery systems as a top global mental health priority. ${ }^{6}$

The call to scale-up global mental health care services in an evidence-based manner requires the systematic development of translational $(\mathrm{T})$ research, including $\mathrm{T} 1, \mathrm{~T} 2$, and T3 studies. T1 research is the evidence foundation, which establishes the efficacy of treatments for a particular target population. T2 activities test the effectiveness of the treatments for the broader local population and then develop practice guidelines. T3 studies address the delivery of evidence-based practices in a manner that is widely accessible, including the needed policy change.
Correspondence: Susan Meffert Department of Psychiatry, University of California, San Francisco,

40I Parnassus Avenue, San Francisco, CA 94143 , USA

$\mathrm{Tel}+\mathrm{I} 4154766100$

Fax + I 4154767404

Email susan.meffert@ucsf.edu 
Conflict- and disaster-affected populations bear the most acute burden of mental disorders, particularly depression and posttraumatic stress disorder (PTSD)..$^{7-10}$ de Jong et al found that the prevalence of PTSD ranged from $15.8 \%$ to $37.4 \%$ across four post-conflict settings. ${ }^{11}$

There has been controversy regarding the treatment of trauma-related mental illness in non-Euro-American cultures, with some critics arguing that the use of diagnoses such as PTSD imposes a potentially harmful sense of victimization upon the narrative of the survivor and draws attention away from the socioeconomic conditions, which contribute to the distress. ${ }^{13-15}$ Indeed, recent data-driven analyses suggest that mental illness among trauma survivors persists despite improvement in socioeconomic conditions. Despite the changes in social, legal, and material conditions that accompanied their asylum and move to the US, a cross-sectional study of 586 Cambodian refugees 20 years after resettlement in Los Angeles revealed a PTSD rate of $62 \%$ and a major depression rate of $51 \% .{ }^{8}$ A recent review of mental health and poverty interventions in LMICs found that such programs had no effect on participants' mental health. ${ }^{16}$

The purpose of this paper is, first, to systematically review the T1 level research for adults in LMICS, and then to present a logic model on which to build the foundation for advanced translational research.

\section{Methods}

\section{Definitions and inclusion/exclusion criteria}

Articles were eligible for inclusion in this study if they were published in English and used quantitative methods to examine the efficacy of the mental health care treatment for adults in LMICs who had been exposed to mass trauma. For the purposes of this paper, populations experiencing mass trauma were defined as those exposed to and/or displaced by armed conflict or natural disaster and refugee populations. Descriptive studies, case reports, comments, editorials, letters, reviews, and studies performed in high-income countries were excluded. Given the relative newness of the field, the limited number of studies, and the wish to be as inclusive as possible, we elected to use broad eligibility criteria that allowed for a diversity of studies with different study designs, treatment modalities, measures, study populations, and sample sizes.

\section{Search strategy}

The review was limited to peer-reviewed articles published in 1980 or after and retrievable from MEDLINE, PSYCHINFO, and CINAHL. For LMIC, the following search terms were used: "developing country," "refugee," "conflict," "postconflict," "global," "low and middle income countries," and "LMIC." For mass trauma, the following search terms were used: "natural disaster," "armed conflict," "war," "displaced," and "refugee." For mental health, the following search terms were used: "mental illness," "psychosocial," "depression," "posttraumatic stress disorder," "PTSD," "substance abuse," and "mental health." For intervention, the following search terms were used: "trial," "care," "services," "treatment," "randomized controlled trial," "RCT," and "intervention." The initial literature search took place from July to August 2009. Additional literature searches took place in July 2012. Therefore, this review includes articles published from January 1980 through July 2012.

\section{Data synthesis}

Studies were grouped by study design: (1) randomized controlled clinical trials, and (2) mental health intervention studies without control and/or randomization.

\section{Data analysis}

Effect sizes were calculated for each controlled trial in order to allow for comparison between studies. The following equation was used:

$$
\text { Cohen's } d=\mathrm{M}_{1}-\mathrm{M}_{2} / \sigma_{\text {pooled }} \text {, }
$$

where $M_{1}=$ mean of the treatment group at the earliest posttreatment time point, $\mathrm{M}_{2}=$ mean of the control group at the earliest posttreatment time point, and $\sigma_{\text {pooled }}=$ pooled standard deviation. $\sigma_{\text {pooled }}$ was calculated as the square root of the average variance:

$$
\sqrt{\left(\mathrm{SD}^{2}+\mathrm{SD} 2^{2}\right) / 2}
$$

where $\mathrm{SD} 1$ = standard deviation of group one and SD2 = standard deviation of group two.

\section{Results}

Seven randomized controlled clinical trials and six clinical trials without control and/or randomization were found. The characteristics of the studies are included in Tables 1 and 2 .

Nine studies were conducted with populations affected by war and four studies addressed survivors of natural disaster. Clinical trials were from a broad diversity of locations. The sample size ranged from 10 to 4000 with a mean of 439 and 


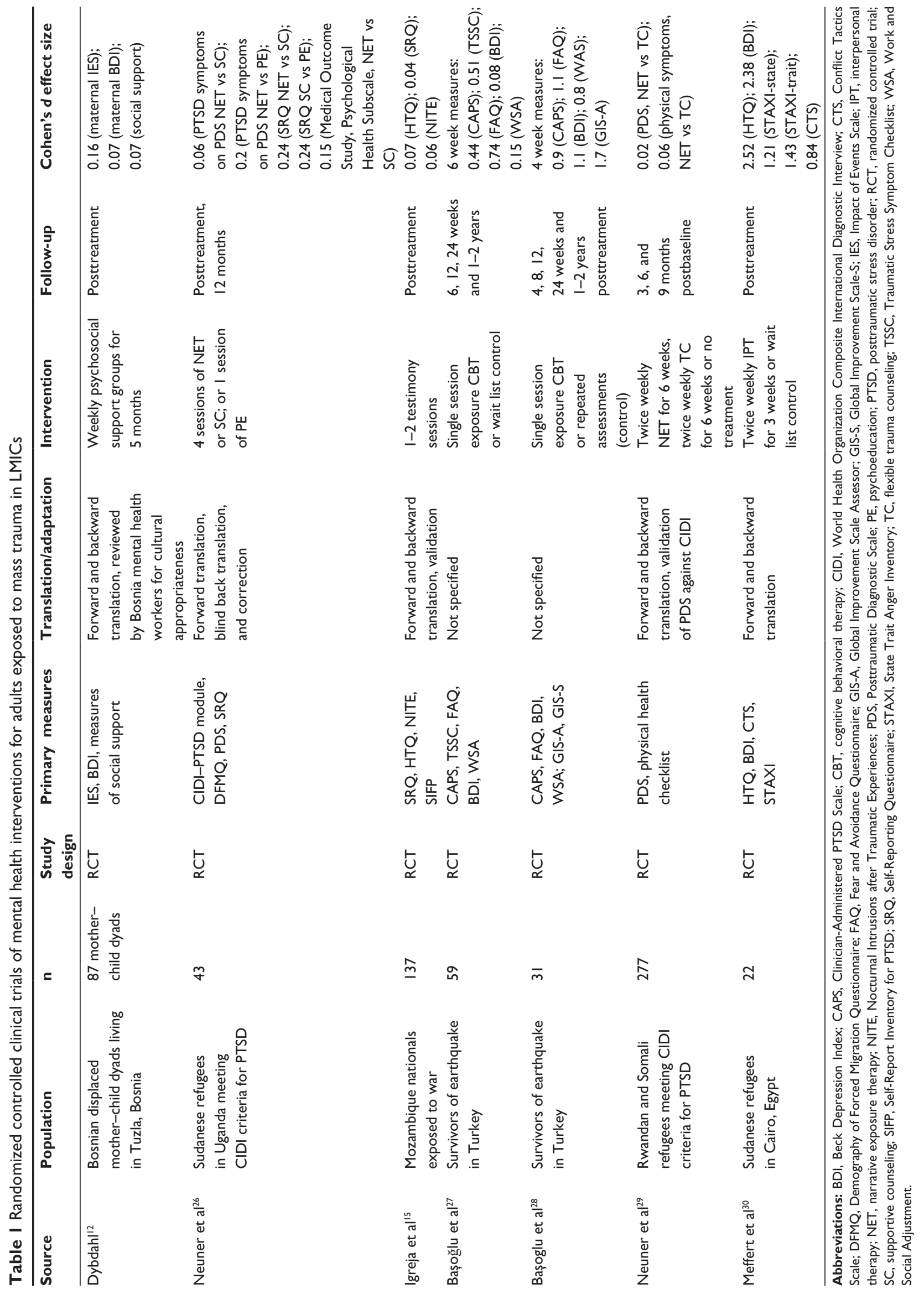




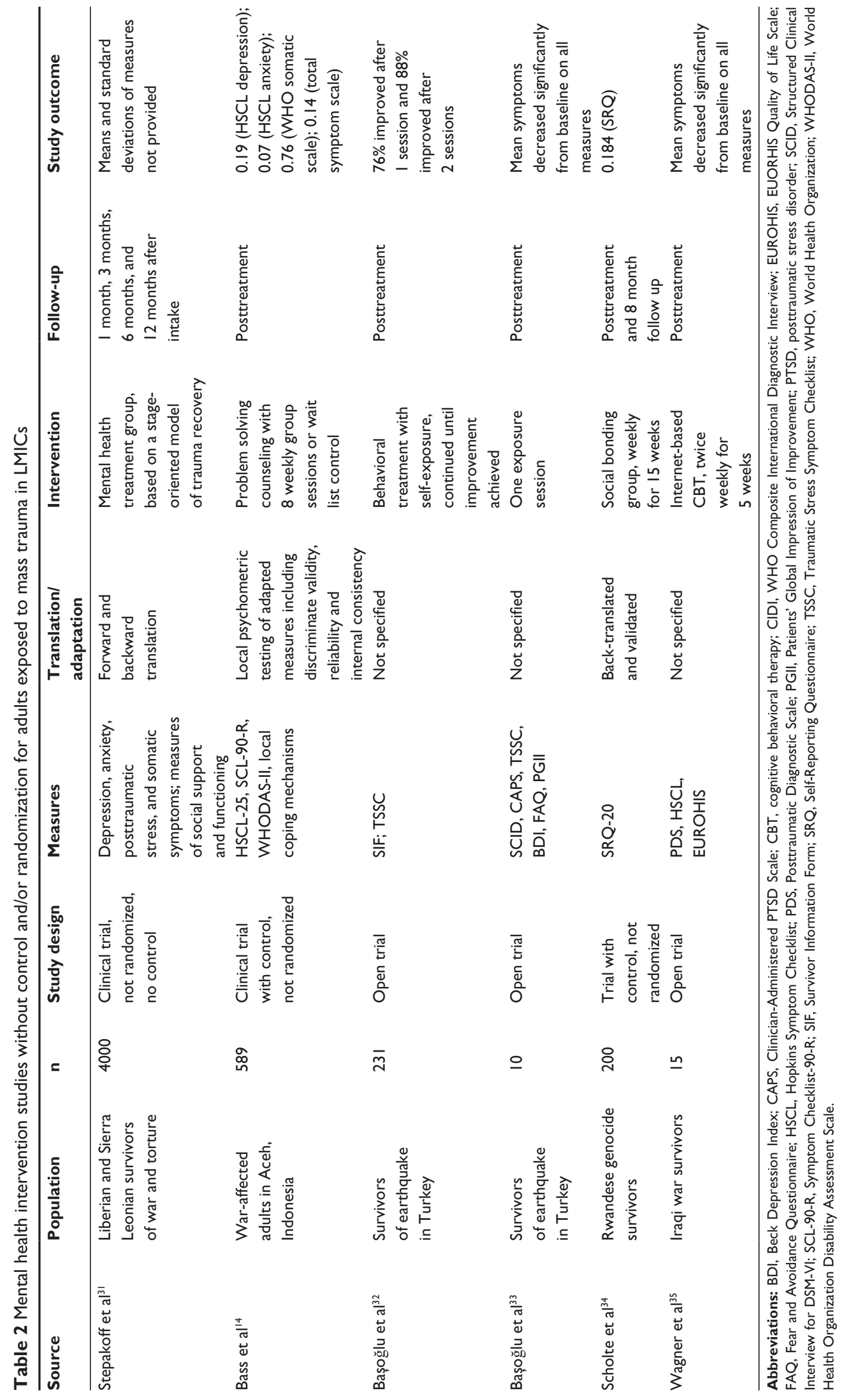


a median of 112. All studies applied baseline measures. The number of outcome/follow-up measure time points varied from one to five and from immediately after posttreatment to 2 years after posttreatment. The measures used in these studies varied on several levels. Some research designs focused on symptoms, while others used mental health diagnoses as the basis for selection and outcome assessment. Most studies used standard symptom checklists to track treatment effect and applied cut-off scores to determine eligibility for inclusion. Four studies used structured clinical interviews such as the Structured Clinical Interview for the Diagnostic and Statistical Manual of Mental Disorders (SCID-DSM-IV), Composite International Diagnostic Interview (CIDI), or Clinician Administered PTSD Scale (CAPS) to determine diagnosis.

Intervention types included interpersonal therapy (IPT), cognitive behavioral therapy, narrative exposure therapy, mixed trauma treatment, testimony therapy, problem-solving counseling, social bonding, and various forms of supportive therapy. None used pharmacologic interventions.

Treatment format varied. In studies where the number of treatment sessions was available, it varied from one to 20 with a mean of 7 and a median of 7 . Four studies tested group modalities and nine tested individual treatments.

Effect sizes were calculated for controlled clinical trials based on the most proximal post-intervention measure. Cohen's $d$ effect sizes ranged from 0.02 to 2.52 .

\section{Discussion}

One of the key findings of this review regards the heterogeneity of the studies, which varied in sample size, popuation, measures, treatment, and follow up. While this makes the comparison of findings between studies difficult, it also clarifies the first steps toward developing a reliable body of T1 research, which should include agreement on the essential aspects of mental health intervention research for mass trauma populations. Below, we discuss differences across the studies and offer a logic model for future T1 research.

\section{Number of studies}

The number of studies seeking to investigate mental health treatments for mass trauma in LIMCs has increased rapidly over the past 10 years, with the majority of publications occurring within the past 5 years. This pattern suggests that the compelling need for global mental health research and service development, stated repeatedly by the WHO and others, is being recognized by researchers and funders.
Given the encouraging trend, we can reasonably expect global mental health research to continue its acceleration, provided that funding keeps pace.

\section{Type of intervention}

The types of mental health interventions used in these studies covered a wide range, from multisession treatment of mother-child dyads to one-time treatment of individuals. ${ }^{12,13}$ The choice of intervention and which, if any, adaptations to apply, often creates a tension between using an established treatment that has been proven to be effective for other populations (most often in high-income countries), and developing a treatment de novo that is tailored to the perceived needs of the target population. One advantage of using established mental health treatments is that they tend to be well-defined, facilitating replication and, to the extent that experience, expression, and emotional healing are similar across cultures, an a priori argument can be made that evidence-based treatments are likely to affect positive change in a new setting. The use of established interventions also makes it easier for other clinicians and researchers to interpret the work and use it for other populations in need.

Few of the studies included or referenced explicit qualitative or ethnographic investigations as being used to inform their choice of mental health intervention. An exception was in Bass et al who described a previous study in the same population that included free listing and qualitative informant interviews. ${ }^{14}$ The results of the study informed the researchers' choice of mental health outcome measures and were subsequently used to adapt measures to assess change in local mental health symptoms. Başoglu et $\mathrm{al}^{13}$ and Igreja et $\mathrm{al}^{15}$ both refer to previous studies, which were used to develop and test the understandings of mental health problems in the study population.

\section{Measures}

These studies used a combination of symptom and diagnostic measures, which illuminate a debate in global mental health research. Some argue that the measures with embedded DSM criteria, such as the SCID, are indexed to Euro-American culture and do not yield accurate diagnostic data when used with other populations. ${ }^{16-19}$ On the other hand, the study results may lack generalizability and be less helpful in advancing the field if standardized diagnostic tools are not used.

\section{Effect size}

The clinical trials reported here demonstrate a wide range of effect sizes (Figure 1). Some of the reasons for the 


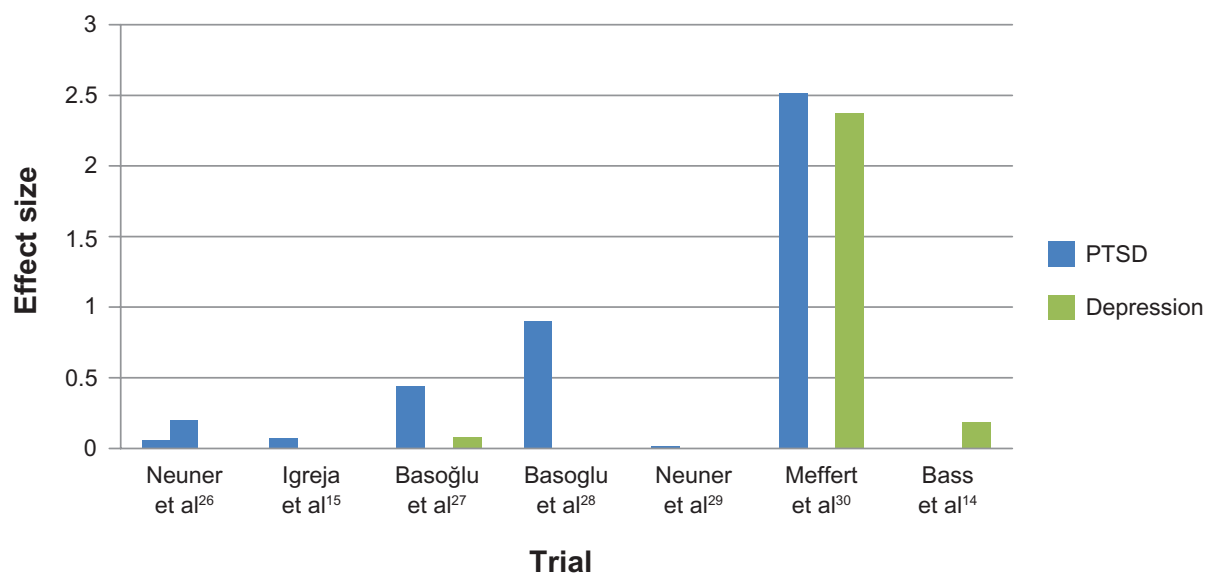

Figure I Effect sizes of mental health treatment trials in LMICs for adults exposed to mass trauma: studies of depression and PTSD. Abbreviations: LMICs, low- and middle-income countries; PTSD, posttraumatic stress disorder.

diversity of effect sizes observed in this review may be independent of the intervention effect. Study populations may vary in their openness to mental health intervention, access to previous mental health treatment, and the resources available to the researchers. For example, in a population for which mental health treatment is both socially accepted and available, the cohort meeting research criteria may have a larger percentage of nonresponders than a similar cohort in a population that has had less exposure to mental health care. Second, populations that have received relatively more mental health care may have less room for improvement in their symptoms, making it more difficult to show a robust effect size with such populations. Furthermore, in lowresource settings, researchers seeking to test sustainable, appropriate treatments are often constrained in their choice of intervention by logistical/material resources, local expertise, and public health demands to use a modality capable of efficiently treating large numbers of people. In some settings, meeting these requirements may require the use of certain treatments or modalities of application (eg, individual versus group) that do not generate optimized effect sizes.

With these caveats in mind, it is interesting to note that IPT showed the highest effect sizes for both depression and PTSD treatment (2.38 and 2.52, respectively). Bolton et al also published a study of IPT with a high effect size for depression in rural Uganda. ${ }^{20}$ Although their study was not included here because it did not focus on trauma survivors, if the same effect size calculation is applied to their data, the Cohen's $d$ for IPT intervention of depression is 1.87. Data are limited, but are consistent with the identification of adapted IPT as a promising mental health intervention for depression and PTSD in LMICs.

\section{Follow-up}

All studies used a post-intervention measure and the majority of the studies used an additional follow-up measure, ranging from 8 weeks to 2 years post-intervention. Follow-up measures are critical for determining the ability of the intervention to affect sustained improvement. Given the occurrence of spontaneous recovery from depression and PTSD, as well as post-intervention relapse, assessing intervention and control groups over time is essential for determining the trajectories of intervention and control.

\section{Global mental health research challenges}

Creating an evidence base for effective global mental health interventions for populations exposed to mass trauma is challenging, partly due to factors that also affect every other health endeavor in LMICs, including scarcity of resources, low levels of expertise, challenging logistics, and the stigmatization of health conditions. Fields such as global mental health, which are heavily influenced by local culture, face the additional challenge that interventions for culturally distinct populations may require additional research: the interaction of culture with emotional experience, expression, and norms means that the indicators of mental illness and the most effective ways of accessing and treating those in need of care must be indexed to the specific community. These caveats make T1 global mental health research challenging. Below, we present a logic model to facilitate the development of T1 interventional research in a manner that is both respectful of the community and builds a foundation for $\mathrm{T} 2$ and $\mathrm{T} 3$ level implementation.

\section{Logic model}

The model offered here is an idealized trajectory (Figure 2) to be used as a methodological guide and adapted as 


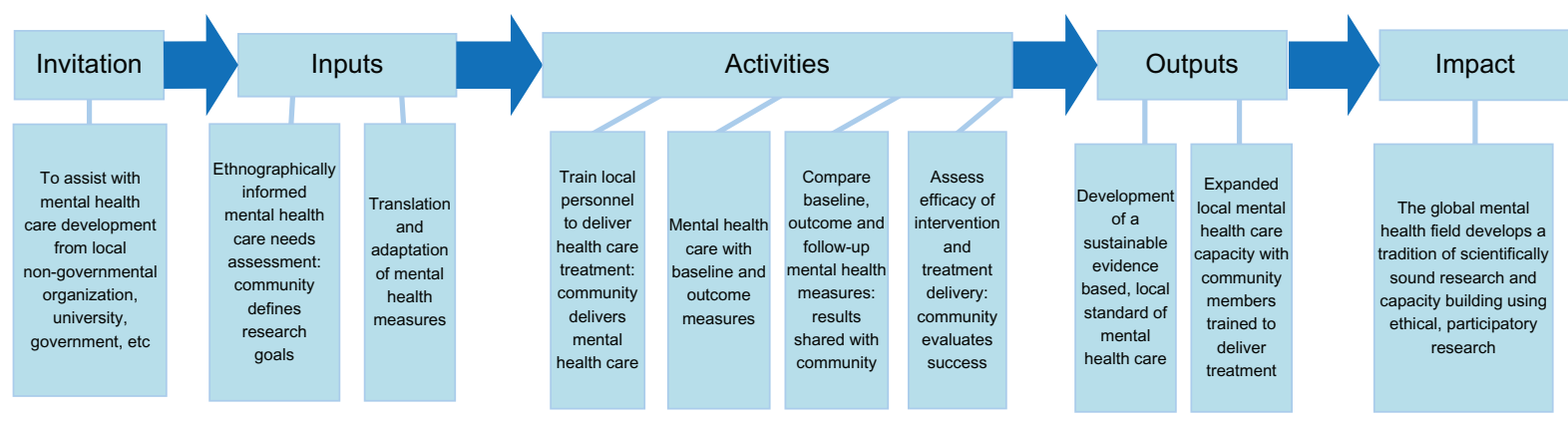

Figure 2 Global mental health research logic model.

circumstances require. We focused this model on the research process, not content; therefore, it can be used for diverse settings with different content demands.

The guiding principles of our model are participatory research methods and scientific rigor. We suggest that if the research involves foreign investigators, those researchers should preferably receive an explicit invitation from a local nongovernmental organization, university, and/or government to assist with evaluating mental health care needs or developing/testing mental health interventions for future programming.

We suggest that research begin with a period of ethnographic work, focused on the determination of the population's own perceptions of their psychosocial health care needs. These needs, among others, may vary widely depending on the circumstances of the traumatic events, the temporal distance from the events, available community resources, and health care efforts. For example, some populations may have undergone the index traumatic event a decade ago, and may still have large amounts of untreated depression and PTSD. For other post-trauma populations, substance abuse and dependence may be the dominant problems.

The goals for the ethnographic period of the work are to learn what, if any, emotional and relationship problems are recognized by the community, what words are used to describe these problems, what methods of coping are used, and what types of problems the community identifies as exceeding their usual coping mechanisms. In conjunction with the last goal, the ethnographic work can include thorough discussions with a cross section of the community regarding what the community hopes to improve with respect to its psychosocial care resources. If needs are identified by the community, then their interest in collaboration with the research team can be discussed. If collaboration is desired, then the benefits, risks, and limitations of the potential research can be reviewed and the details of the community's wishes can be delineated. With the active involvement of the community, a collaborative plan for addressing these wishes, along with a clear articulation of the goals for each phase of the research, and the possible programmatic outcomes of the research can all be developed. With the goal of selecting an evidence-based intervention, which is both acceptable to and appropriate for the community, the options for mental health intervention can be reviewed with the community. The concluding section of the qualitative work includes the selection, translation, and adaptation of measures to be used in the quantitative phase of the research. ${ }^{21-24}$ It is important to note that it is not always possible to conduct and analyze a lengthy ethnographic study of the target population, particularly in circumstances of acute need. If necessary, qualitative needs assessment studies can use focused ethnographic methods to make rapid estimates of needs in a matter of weeks. ${ }^{23}$

We suggest that the research plan be implemented in the context of regular dialogue with the community and include involvement of community members as the staff and supervisors of the research program. If the research uses a clinical trial design, local personnel should be trained to deliver the intervention. Intervention adherence rating procedures need to be developed to ascertain intervention fidelity. The target outcomes of the study participants are ideally measured with symptom and functioning measures at baseline, mid-point, end-point, and/or follow-up(s), as indicated.

As much as possible, publications of global mental health research should adhere to defined standards for reporting of clinical trials, such as those described by the CONSORT guidelines. ${ }^{25}$

In addition to such guidelines, the reporting of global mental health intervention trials may benefit from a description of the mental health care needs assessment preceding the clinical trial; a report of how study measures were adapted, translated, and/or validated for the local population; and the method(s) used to involve the local community and contribute to capacity building. 


\section{Troubleshooting and adaptations}

In practice, the logic model must often be adapted according to resource limitations or as difficulties arise in the course of research. For example, the team may not have the financial means, personnel, or expertise to meet the community's stated mental health care needs. In such cases, a graduated approach may be required in which steps toward the community's goals are matched with the team's current capacity and additional resources are obtained to meet long-term objectives.

\section{Conclusion}

In summary, the strong call to develop global mental health implementation research for populations affected by mass trauma is based on compelling epidemiological evidence of a high disease burden. The publication of studies in this area has increased over the past 5 years and promises to continue expanding. We present the logic model described above as an approach for refining current methods of T1 research and preparing for the development of robust $\mathrm{T} 2$ and $\mathrm{T} 3$ research in the years ahead.

\section{Limitations}

As noted in our methods section, given the relatively early stage of this research field and the limited number of studies, we prioritized inclusion of as many studies as possible. We acknowledge that this approach allows for heterogeneity, which makes comparison between studies difficult. Nonetheless, this heterogeneity is indeed a key finding of our study, as it may represent the central hurdle for developing a T1 research base. We acknowledge that our logic model is general and may lack specific guidance. In the interest of creating a tool that can be used with many populations, we constructed an adaptable logic model that we hope will assist with achieving greater research consistency, while allowing for cultural variation. As noted in the methods section, this review included only Englishlanguage publications. This restriction may have led to the exclusion of relevant studies that were not published in English. We acknowledge that effect sizes for small samples must be interpreted with caution. We emphasize that this is a review of literature in a nascent field and is intended to promote additional $\mathrm{T} 1$ research, not to identify effective interventions.

\section{Acknowledgments}

We would like to thank Drs Thomas Neylan, Kevin Delucchi, Charles Marmar, and Robert Brooks for reviewing earlier versions of this manuscript.

\section{Disclosure}

The authors report no conflicts of interest in this work.

\section{References}

1. Lancet Global Mental Health Group, Chisholm D, Flisher AJ, et al. Scale up services for mental disorders: a call to action. Lancet. 2007;370: 1241-1252.

2. Lopez AD, Mathers CD, Ezzati M, Jamison DT, Murray CJL. Global Burden of Disease and Risk Factors. Oxford: Oxford University Press; 2006.

3. Murray CJL, LopezAD. The Global Burden of Disease: A Comprehensive Assessment of Mortality and Disability from Diseases, Injuries, and Risk Factors in 1990 and Projected to 2020. London: Harvard University Press; 1996.

4. Saxena S, Thornicroft G, Knapp M, Whiteford H. Resources for mental health: scarcity, inequity, and inefficiency. Lancet. 2007;370:878-889.

5. Patel V, Garrison P, de Jesus Mari J, Minas H, Prince M, Saxena S; Advisory group of the Movement for Global Mental Health. The Lancet's series on global mental health: 1 year on. Lancet. 2008;372: 1354-1357.

6. Tomlinson M, Rudan I, Saxena S, Swartz L, Tsai AC, Patel V. Setting priorities for global mental health research. Bull World Health Organ. 2009;87:405-484

7. Fazel M, Wheeler J, Danesh J. Prevalence of serious mental disorder in 7000 refugees resettled in western countries: a systematic review. Lancet. 2005;365:1309-1314.

8. Marshall GN, Schell TL, Elliott MN, Berthold SM, Chun CA. Mental health of Cambodian refugees 2 decades after resettlement in the United States. JAMA. 2005;294:571-579.

9. Onyut L, Neuner F, Ertl V, Schauer E, Odenwald M, Elbert T. Trauma, poverty and mental health among Somali and Rwandese refugees living in an African refugee settlement - an epidemiological study. Conf Health. 2009;3:6.

10. Steel Z, Chey T, Silove D, Marnane C, Bryant RA, van Ommeren M. Association of torture and other potentially traumatic events with mental health outcomes among populations exposed to mass conflict and displacement: a systematic review and meta-analysis. JAMA. 2009;302:537-549.

11. de Jong JT, Komproe IH, Van Ommeren M. Common mental disorders in postconflict settings. Lancet. 2003;361:2128-2130.

12. Dybdahl R. Children and mothers in war: an outcome study of a psychosocial intervention program. Child Dev. 2001;72:1214-1230.

13. Başoglu M, Salcioglu E, Livanou MA. Randomized controlled study of single-session behavioural treatment of earthquake-related post-traumatic stress disorder using an earthquake simulator. Psychol Med. 2007;37:203-213.

14. Bass J, Poudyal B, Tol W, Murray L, Nadison M, Bolton P. A controlled trial of problem-solving counseling for war-affected adults in Aceh, Indonesia. Soc Psychiatry Psychiatr Epidemiol. 2012;47:279-291.

15. Igreja V, Kleijn WC, Schreuder BJN, Van Dijk JA, Verschuur M. Testimony method to ameliorate post-traumatic stress symptoms: Community-based intervention study with Mozambican civil war survivors. Br J Psychiatry. 2004;184:251-257.

16. Young A. The Harmony of Illusions: Inventing Post-Traumatic Stress Disorder. Princeton, NJ: Princeton University Press; 1997.

17. Summerfield D. A critique of seven assumptions behind psychological trauma programmes in war-affected areas. Soc Sci Med. 1999;48: 1449-1462.

18. Summerfield DA. Legacy of war: beyond "trauma" to the social fabric. Lancet. 1997;349:1568.

19. Pupavac V. Psychosocial interventions and the demoralization of humanitarianism. J Biosoc Sci. 2004;36:491-504.

20. Bolton P, Bass J, Neugebauer R, et al. Group interpersonal psychotherapy for depression in rural Uganda: a randomized controlled trial. JAMA. 2003;289:3117-3124. 
21. Bolton P. Cross-cultural validity and reliability testing of a standard psychiatric assessment instrument without a gold standard. J Nerv Ment Dis. 2001;189:238-242.

22. Berry JW, Poortinga YH, Pandey J, editors. Handbook of Cross-cultural Psychology Volume 1 (2nd edition): Theory and Method. Boston: Allyn \& Bacon, Inc; 1996.

23. de Jong JTVM, Van Ommeren M. Toward a culture-informed epidemiology: combining qualitative and quantitative research in transcultural contexts. Transcult Psychiatry. 2002;39:422-433.

24. Smit J, Van den Berg CE, Bekker LG, Seedat S, Stein DJ. Translation and cross-cultural adaptation of a mental health battery in an African setting. Afr Health Sci. 2006;6:215-222.

25. Moher D, Schulz KF, Altman DG. The CONSORT statement: revised recommendations for improving the quality of reports of parallel-group randomised trials. Lancet. 2001;357:1191-1194.

26. Neuner F, Schauer M, Klaschik C, Karunakara U, Elbert T. A comparison of narrative exposure therapy, supportive counseling, and psychoeducation for treating posttraumatic stress disorder in an African refugee settlement. J Consult Clin Psychol. 2004;72:579-587.

27. Başoğlu M, Salcioğlu E, Livanou M, Kalender D, Acar G. Single-session behavioral treatment of earthquake-related posttraumatic stress disorder: a randomized waiting list controlled trial. J Trauma Stress. 2005;18:1-11.

28. Başoglu M, Salcioglu E, Livanou M. A randomized controlled study of single-session behavioural treatment of earthquake-related post-traumatic stress disorder using an earthquake simulator. Psychol Med. 2007;37:203-213.
29. Neuner F, Onyut PL, Ertl V, Odenwald M, Schauer E, Elbert T. Treatment of posttraumatic stress disorder by trained lay counselors in an African refugee settlement: a randomized controlled trial. J Consult Clin Psychol. 2008;76:686-694.

30. Meffert SM, Abdo AO, Alla OA, et al. Sudanese refugees in Cairo, Egypt: a randomized controlled trial of interpersonal psychotherapy for trauma, depression and interpersonal violence. Psychol Trauma. May 2, 2011.

31. Stepakoff S, Hubbard J, Katoh M, et al. Trauma healing in refugee camps in Guinea: a psychosocial program for Liberian and Sierra Leonean survivors of torture and war. Am Psychol. 2006;61:921-932.

32. Başoğlu M, Livanou M, Salcioğlu E, Kalender D. A brief behavioural treatment of chronic post-traumatic stress disorder in earthquake survivors: results from an open clinical trial. Psychol Med. 2003;33:647-654.

33. Başoğlu M, Livanou M, Salcioğlu E. A single session with an earthquake simulator for traumatic stress in earthquake survivors. Am J Psychiatry. 2003;160:788-790.

34. Scholte WF, Verduin F, Kamperman AM, Rutayisire T, Zwinderman AH, Stronks K. The effect on mental health of a large scale psychosocial intervention for survivors of mass violence: a quasi-experimental study in Rwanda. PLoS One. 2011;6(8):e21819.

35. Wagner B, Schulz W, Knaevelsrud C. Efficacy of an Internet-based intervention for posttraumatic stress disorder in Iraq: a pilot study. Psychiatry Res. 2012;195(1-2):85-88.
Open Access Journal of Clinical Trials

\section{Publish your work in this journal}

The Open Access Journal of Clinical Trials is an international, peerreviewed, open access journal publishing original research, reports, editorials, reviews and commentaries on all aspects of clinical tria design, management, legal, ethical and regulatory issues, case record form design, data collection, quality assurance and data auditing

\section{Dovepress}

methodologies. The manuscript management system is completely online and includes a very quick and fair peer-review system, which is all easy to use. Visit http://www.dovepress.com/testimonials.php to read real quotes from published authors. 